\title{
MAC Controller for Path Tracking of a Wheeled Mobile Robot in the Presence of Skidding Effects
}

\author{
Yuanliang Zhang ${ }^{1}$, Ji Hyoung Ryu ${ }^{2 *}$ and Kil To Chong ${ }^{3}$ \\ ${ }^{1}$ School of Automation, Southeast University \\ ${ }^{2}$ Electronics and Telecommunications Research Institute \\ ${ }^{3}$ School of Electronics and Information, Chonbuk National University \\ ${ }^{1}$ zhangyuanl@hhit.edu.cn, ${ }^{2 *}$ jihyoung@etri.re.kr, ${ }^{3}$ kitchong@jbnu.ac.kr
}

\begin{abstract}
This paper proposed a model algorithm control (MAC) method for path tracking control of a differentially-steered wheeled mobile robot (WMR) considering the presence of disturbances that violate the nonholonomic constraints. MAC is a one-step-ahead predictive controller in which the control law is obtained by minimizing the output error. The design of MAC controller is based on the discretized form of the control system. In this paper a time discretization method using the Taylor series and zero-order-hold ( $\mathrm{ZOH})$ assumption was proposed to discetize the continuous model of the WMR. Then MAC controller was designed to perform the tracking control for the mobile robot basing on the WMR's discretized model. The presence of skidding effects which violates the nonholonomic constraints was considered during simulations and experiments done to verify the performance and feasibility of the proposed control strategy.
\end{abstract}

Keywords: model algorithm control; path tracking control; skidding effects; wheeled mobile robot

\section{Introduction}

Nonholonomic systems commonly occur in finite dimensional mechanical systems where constraints are imposed on the non-integrable motions (the constraints cannot be written as time derivatives of a function of the generalized coordinates). There are numerous examples of nonholonomic systems which are of substantial engineering interest. The constraints involved are based on the assumption that there is no substantial slipping of wheels in the case of a differential driven WMR.

The control of wheeled mobile robots has received a lot of attention from the control and robotics communities due to their theoretical control design difficulties and the wide range of applications [1-3]. Posture stabilization and path tracking are the two fundamental states when controlling a WMR. Posture stabilization's purpose is to stabilize the WMR to a reference point while path tracking is to make the WMR to follow a reference path. Posture stabilization is of great theoretical interest where good performance is very difficult to obtain and its solution usually does not give great practical advantages [4]. Good tracking ability is essential for the WMRs since most of the tasks involve tracking a predefined collision-free path and/ or a detected path in realtime. It is for this reason that the problem of path tracking control of the WMRs is thoroughly investigated in this literature.

Path tracking control is easier to achieve than posture stabilization for the WMRs. This comes from the assumption that the wheel makes perfect contact with the ground and there is no skidding during the running process. It results in the nonholonomic constraints in which not all velocities are possible at a certain moment. This is a fundamental motion

${ }^{*}$ Corresponding Author 
control problem and has been thoroughly investigated in the robotic domain [5-10]. A linear tracking controller for mobile robots was reported by [11] and [12]. However, the controller works only when the linear velocity is not zero for the linear model approach. It is difficult to control the mobile robot to keep tracking in a specified path and stop at a specific point, thus nonlinear controllers for this class of systems have been extensively studied.

Zenon and Marcin [13] proposed a discrete algorithm for a tracking control of a WMR using an advanced Adaptive Critic Design. Lim, et al., [14] presented a nonlinear model predictive tracking control scheme for a wheeled nonholonomic unmanned ground vehicle. Dinh, et al., [15] presented a differential sliding mode tracking controller for trajectory tracking of the three-wheeled mobile platform. Chen et al., [16] proposed a complete control law comprising a kinematic control and a sliding-mode dynamic control for the trajectory tracking control of nonholonomic WMRs. The assumptions of the nonholonomic constraints are still ideally satisfied even though the disturbance or parameter variation influences were considered in the above-mentioned papers. However, the ideal, pure rolling, and no-slipping assumption for the WMRs are unrealistic in the real-world and it should be noted that they will be violated in practical applications. The sliding effects were modeled considering the slipping for non-straight trajectories by [17] and [18].

In this paper, we presented the problem of controlling a WMR subject to skidding effects where the ideal nonholonomic constraints of pure rolling and non-slipping are violated. This could be caused by sliding, deformability, or flexibility of the wheels. Model Algorithm Control (MAC) method for the tracking control of the WMR is proposed. MAC is a one-step-ahead predictive controller in which the control law is obtained by minimizing the output error at time $(k+\sigma) T$, where $k T$ is the time of $k$ sampling intervals and $\sigma T$ indicates the time delay. It basically involves an impulse response model for system representation and prediction, a reference path and an optimality criterion. The main idea of the MAC strategy is to predict the deviation of the future system outputs from the reference path based on the model, define an optimality criterion that reflects the deviations, and obtain an optimality control strategy to minimize the criterion over a certain horizon in the future.

This paper is organized into the following sections: Section 2 presents the kinematic model of the WMR, Section 3 introduces a nonlinear system discretization method using the Taylor series and $\mathrm{ZOH}$ assumption, Section 4 presents the MAC controller synthesis for the tracking control of the WMR, Section 5 demonstrates the performance of the proposed MAC controller using computer simulations and experiment, and Section 6 presents the conclusion of this study.

\section{Mathematical Model of the Wheeled Mobile Robot}

Let's consider a nonholonomic WMR with two differentially steered wheels, as shown in Figure 1. It shows a geometrical model of the WMR including the necessary variables to obtain the kinematic model. This WMR has two driving wheels (radius $r$ ) and one caster. Point $H\left(x_{H}, y_{H}\right)$ defines the intersection of the axis of symmetry with the driving wheel axis and is assumed to be the origin of the coordinate frame $\left\{X_{H}, Y_{H}\right\}$. Point $C\left(x_{c}, y_{c}\right)$ is the center of mass of the mobile robot. Length $c$ is the distance between point $H$ and point $C$ and $l$ is the length of the rear wheel axis. 


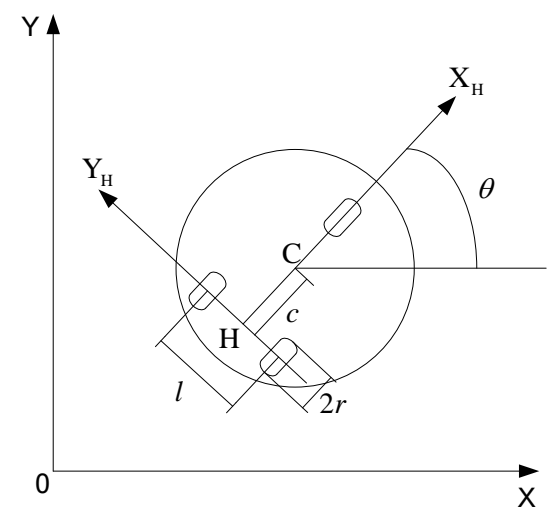

Figure 1. Sketch of a Nonholonomic WMR

For this kind of WMR, there are three constraints according to [19]. The first one is that the robot must move in the direction of the axis of symmetry.

$\dot{x}_{H} \sin \theta-\dot{y}_{H} \cos \theta=0$

The other two constraints are the rolling constraints, in which the left and right driving wheels do not slip.

$\dot{x}_{H} \cos \theta+\dot{y}_{H} \sin \theta+l \dot{\theta} / 2-r \dot{\varphi}_{r}=0$

$\dot{x}_{H} \cos \theta+\dot{y}_{H} \sin \theta-l \dot{\theta} / 2-r \dot{\varphi}_{l}=0$

where the variable $\theta$ is the angle between the direction of the WMR and the $X$ axis while $\varphi_{r}$ and $\varphi_{l}$ are the angular displacements of the right and left driving wheels respectively.

Assume that $\omega(t)$ and $v(t)$ are the angular and the displacement velocities, respectively. The WMR can be described by the following kinematic model:

$$
\left\{\begin{array}{l}
\dot{x}_{H}=v(t) \cos (\theta) \\
\dot{y}_{H}=v(t) \sin (\theta) \\
\dot{\theta}=\omega(t)
\end{array}\right.
$$

Eq. (4) corresponds to the hypothesis of "pure rolling and non-slipping condition" for the WMR. Assume that a bounded disturbance $\eta(t),|\eta(t)| \leq \rho$, where $\eta(t)$ is the quality measuring the violation of the nonholonomic constraints associated to Eq. (4) $[20] . \rho$ is a known constant. Then Eq. (1) becomes:

$\dot{x}_{H} \sin \theta-\dot{y}_{H} \cos \theta=\eta(t)$

The kinematic model of the mobile robot with unmatched disturbances becomes: 


$$
\left\{\begin{array}{l}
\dot{x}_{H}=v(t) \cos (\theta)+\eta(t) \sin (\theta) \\
\dot{y}_{H}=v(t) \sin (\theta)-\eta(t) \cos (\theta) \\
\dot{\theta}=\omega(t)
\end{array}\right.
$$

\section{Time Discretization Method for Nonlinear Control System}

The time discretization method presented in this paper can provide a relatively more exact discrete-time model for a continuous nonlinear system. The general multi-input nonlinear system in state-space form can be represented as follows:

$$
\begin{aligned}
& \frac{d x(t)}{d t}=f(x(t))+\sum_{i=1}^{a} g_{i}(x(t)) u_{i}\left(t_{i}\right)=f(x(t)) \\
& +u_{1}(t) g_{1}(x(t))+u_{2}(t) g_{2}(x(t))+\cdots+u_{a}(t) g_{a}(x(t)), \\
& y=h(x(k))
\end{aligned}
$$

Where $x \in X \subset R^{n}$ represents the system state, $u_{i} \in R(i=1 \ldots a)$ is an input variable, and $f(x)$ and $g_{i}(x)(i=1 \ldots a)$ are nonlinear functions for $x$. In addition, the $\mathrm{ZOH}$ is assumed for a fixed sampling period: $u_{i}(t)=u_{i}(k T) \equiv u_{i}(k)=$ constant, $k T \leq t<k T+T$. Here $T$ is the sampling period. The expressions of the states for each sampling period can be obtained using the Taylor series. The general discretized form of the multi-input nonlinear system can be derived as follows:

$$
x(k+1)=x(k)+\sum_{l=1}^{M} A^{l}\left(x(k), u_{1}(k), u_{1}(k) \ldots, u_{a}(k)\right) \frac{T^{l}}{l !}
$$

where $x(k)$ is the value of a state vector of $x$ at $t=t_{k}=k T, M$ is truncation order of the Taylor series. $A^{[l]}(x, u)$ is defined recursively by using Eq. (9).

$$
\begin{aligned}
& A^{[1]}(x, u)=f(x)+u_{1} g_{1}(x)+u_{2} g_{2}(x)+\cdots+u_{a} g_{a}(x) \\
& \cdots \\
& A^{[l+1]}(x, u)=\frac{\partial A^{[l]}(x, u)}{\partial x}\left(f(x)+u_{1} g_{1}(x)+u_{2} g_{2}(x)+\cdots+u_{a} g_{a}(x)\right), \\
& l=1,2,3, \ldots
\end{aligned}
$$

Define the function $\Phi_{T}^{M}$ as follows:

$$
x(k+1)=\Phi_{T}^{M}\left(x(k), u_{1}(k), u_{2}(k), \ldots, u_{a}(k)\right)
$$

where the function $\Phi_{T}^{M}$ depends on the sampling period of $T$ and truncation order of $M$.

Let $\mathbf{U}(k)=\left[\begin{array}{llll}u_{1}(k) & u_{2}(k) & \ldots & u_{a}(k)\end{array}\right]^{T}$ and the sampled-data representation can be written in a more compact form as: 


$$
\begin{aligned}
& x(k+1)=\Phi_{T}^{M}(x(k), \mathbf{U}(k)), \\
& y=h(x(k)) .
\end{aligned}
$$

\section{Model Algorithm Control for Nonlinear System}

Consider the nonlinear systems described by a discrete-time state-space form:

$x_{m}(k+1)=\Phi\left[x_{m}(k), u(k)\right]$,

$y_{m}(k)=h\left[x_{m}(k)\right]$.

where the subscript $m$ is added to indicate estimates of $x$ and $y$ obtained in the model simulation and differentiate the simulated $y_{m}$ from the measured output $y$. $x_{m}=\left[x_{1}, x_{2}, \ldots, x_{n}\right]$ denotes the vector of state variables, $u=\left[u_{1}, u_{2}, \ldots . . u_{a}\right]$ denotes the manipulated inputs, and $y_{m}=\left[y_{1}, y_{2}, \ldots, y_{n}\right]$ represents the output to be controlled. It is assumed that $x_{m} \in X \subset R^{j}$ and $u \in U \subset R^{i}$, where $X$ and $U$ are open-connected sets that contain the origin (that is, the nominal equilibrium point). $\Phi(x, u)$ is an analytic vector function on $X \times U$, and $h(x)$ is an analytic scalar function on $X$.

Suppose that system (12) has the relative order, $\sigma$, which is the smallest number of sampling periods after which the manipulated input $u$ affects the output, $y$. This means that:

$$
\begin{aligned}
& {\left[\frac{\partial h(x)}{\partial x}\right]\left[\frac{\partial \Phi(x, u)}{\partial x}\right]^{p}\left[\frac{\partial \Phi(x, u)}{\partial u}\right]=0, p=0,1, \ldots, \sigma-2} \\
& {\left[\frac{\partial h(x)}{\partial x}\right]\left[\frac{\partial \Phi(x, u)}{\partial x}\right]^{\sigma-1}\left[\frac{\partial \Phi(x, u)}{\partial u}\right] \neq 0}
\end{aligned}
$$

Online simulation of the model described by Eq. (12) can be used to predict the future changes in the output $y$ as follows:

$$
\begin{aligned}
& y_{m}(k+1)-y_{m}(k)=h^{1}\left[x_{m}(k)\right]-h\left[x_{m}(k)\right] \\
& y_{m}(k+2)-y_{m}(k)=h^{2}\left[x_{m}(k)\right]-h\left[x_{m}(k)\right] \\
& \vdots \\
& y_{m}(k+\sigma-1)-y_{m}(k)=h^{\sigma-1}\left[x_{m}(k)\right]-h\left[x_{m}(k)\right] \\
& y_{m}(k+\sigma)-y_{m}(k)=h^{\sigma-1}\left[\Phi\left[x_{m}(k), u(k)\right]-h\left[x_{m}(k)\right],\right.
\end{aligned}
$$

and the following notation is used:

$$
\left\{\begin{array}{l}
h^{0}(x)=h(x) \\
h^{p}(x)=h^{p-1}[\Phi(x, u)], p=1, \ldots, \sigma-1
\end{array}\right.
$$

Here, take into account Eq. (13) that can be represented in the form: 


$$
\begin{aligned}
& \frac{\partial}{\partial u} h^{p}[\Phi(x, u)]=\left[\frac{\partial h(x)}{\partial x}\right]\left[\frac{\partial \Phi(x, u)}{\partial x}\right]^{p}\left[\frac{\partial \Phi(x, u)}{\partial u}\right]=0, p=0,1, \ldots, \sigma-2, \\
& \frac{\partial}{\partial u} h^{\sigma-1}[\Phi(x, u)]=\left[\frac{\partial h(x)}{\partial x}\right]\left[\frac{\partial \Phi(x, u)}{\partial x}\right]^{\sigma-1}\left[\frac{\partial \Phi(x, u)}{\partial u}\right] \neq 0
\end{aligned}
$$

Furthermore, the following relations will hold:

$$
\left\{\begin{array}{l}
y(k+p)=h^{p}[x(k)], p=0, \ldots, \sigma-1 \\
y(k+\sigma)=h^{\sigma-1}\{\Phi[x(k), u(k)]\} .
\end{array}\right.
$$

With a finite relative order, $\sigma$, Eq. (17), implies that the algebraic equation

$$
h^{\sigma-1}[\Phi(x, u)]=y
$$

is locally solvable in $u$. The corresponding implicit function will be denoted by:

$$
u=\Psi_{0}(x, y)
$$

and is assumed to be well-defined and unique on $X \times h(X)$.

When these predicted changes shown in Eq. (14) are added to the measured output signal, $y(k)$, one obtains the following closed-loop predictions of the output:

$$
\begin{aligned}
& \hat{y}(k+1)=y(k)+h^{1}\left[x_{m}(k)\right]-h\left[x_{m}(k)\right] \\
& \hat{y}(k+2)=y(k)+h^{2}\left[x_{m}(k)\right]-h\left[x_{m}(k)\right] \\
& \ldots \\
& \hat{y}(k+\sigma-1)=y(k)+h^{\sigma-1}\left[x_{m}(k)\right]-h\left[x_{m}(k)\right] \\
& \hat{y}(k+\sigma)=y(k)+h^{\sigma-1}\left[\Phi\left[x_{m}(k), u(k)\right]\right]-h\left[x_{m}(k)\right]
\end{aligned}
$$

where $\hat{y}(k)$ represents a prediction of the output $y(k)$.

At every time step, the control computer can calculate the output prediction using Eq. (20), driven by $u(k)$ and $y(k)$, where $x_{m}(k)$ is obtained by online simulation of the state equations of Eq. (12): $x_{m}(k+1)=\Phi\left[x_{m}(k), u(k)\right]$.

The question that arises is what would be the best choice of $u(k)$ to obtain a desirable output response after $\sigma$ time steps. If $u(k)$ is chosen so that $\hat{y}(k+\sigma)$ is exactly the set-point value, $y_{s p}$, this would clearly create a non-robust situation, since the output can be seriously affected by disturbances or system errors. Instead, one can request $\hat{y}(k+\sigma)$ to be in the right direction and cover a fraction of the "distance" between $\hat{y}(k+\sigma-1)$ and the set-point value. In other words, one can define a desirable value, $y_{d}$, of the output at the $(k+\sigma)$ th time step by:

$y_{d}(k+\sigma)=(1-\varepsilon) y_{s p}+\varepsilon \hat{y}(k+\sigma-1)$ 
where $\varepsilon$ is a tunable filter parameter such that $0<\varepsilon<1$. Clearly, $\varepsilon \rightarrow 0$ corresponds to $y_{d}(k+r) \rightarrow y_{s p}(k)$, and therefore, will try to force the output to go to the set point as soon as possible, whereas $\varepsilon \rightarrow 1$ corresponds to $y_{d}(k+r) \rightarrow \hat{y}(k+r-1)$, leaving the output unaffected. An intermediate choice of $\varepsilon$ that corresponds to a desirable value of the output between $y_{s p}$ and $\hat{y}(k+\sigma-1)$ tries to bridge the gap to a certain extent. There is not a criterion to choose $\varepsilon$. It depends on the characteristics of the system to be controlled and the control requirement. If the target of control system is to make the system output follow the reference output quickly $\varepsilon$ should be chosen as a small value; on the other hand if the target of control system is to make the system output follow the reference output gently with a small input a big $\varepsilon$ can be used. Eq. (21) is referred to as the "reference trajectory" in the MAC literature.

Once the reference trajectory has been specified, the question then becomes how to choose the control input $u(k)$ so that $\hat{y}(k+r)$ will match $y_{d}(k+r)$. This can be formulated as an optimization problem:

$$
\min _{u(k)}\left[y_{d}(k+\sigma)-\hat{y}(k+\sigma)\right]^{2}
$$

Considering Eqs. (20) and (21), this becomes

$$
\min _{u(k)}\left\{(1-\varepsilon) e(k)-h^{\sigma-1}\left\{\Phi\left[x_{m}(k), u(k)\right]\right\}+\varepsilon h^{\sigma-1}\left[x_{m}(k)\right]+(1-\varepsilon) h\left[x_{m}(k)\right]\right\}^{2}
$$

where $e(k)=y_{s p}(k)-y(k)$.

In the absence of input constraints, this minimization problem is trivially solvable. Input $u(k)$ is the solution of the nonlinear algebraic equation:

$$
\begin{aligned}
& h^{\sigma-1}\left\{\Phi\left[x_{m}(k), u(k)\right]\right\}=\zeta\left(x_{m}(k), e(k)\right) \\
& \text { where } \zeta(x, e)=\varepsilon h^{\sigma-1}[x]+(1-\varepsilon)(h[x]+e) .
\end{aligned}
$$

Recalling the definition of $\Psi_{0}$ (Eq. 19), the solution can be represented as:

$$
u(k)=\Psi_{0}\left\{x_{m}(k), \zeta\left(x_{m}(k), e(k)\right)\right\}
$$

Therefore, the derived control law is given by Eq. (25).

The MAC control method described in this section is applied to construct a controller synthesis for tracking control of the mobile robot described in Section 2. The Eq. (6) is used as the mobile robot model. For simple we can write Eq. (6) as the following simple form.

$$
\dot{\mathbf{X}}_{m}(t)=F\left(\mathbf{X}_{m}(t)\right)+G\left(\mathbf{X}_{m}(t)\right) \mathbf{U}(t)+H\left(\mathbf{X}_{m}(t)\right) \eta(t)
$$

where $\mathbf{X}_{m}=\left[\begin{array}{lll}x_{H} & y_{H} & \theta\end{array}\right]^{T}, \mathbf{U}=\left[\begin{array}{ll}v & \omega\end{array}\right]^{T}$ and $|\eta| \leq \rho$. 
By employing the time discretization method presented in Section 3 the sampleddata representation of the wheeled mobile robot's model can be obtained. Here the discrete time model is assumed as:

$$
\mathbf{X}_{m}(k+1)=\Phi\left(\mathbf{X}_{m}(k), \mathbf{U}(k), \eta(k)\right)
$$

Then just like the description of this section we can get:

$$
\mathbf{U}(k)=\Psi_{0}\left(\zeta\left(\mathbf{X}_{m}(k), \mathbf{E}(k)\right), \eta(k)\right)
$$

After designing the "reference trajectory" we can obtain the control inputs like the Eq. (25).

Theorem 1: Consider the model of the mobile robot described in Eq. (27). If $\mathbf{U}=\Psi_{0}(\mathbf{X}, \eta)$ is well-defined and unique, then the closed loop MAC control system is stable.

Proof: The Lyapunov function is usually used for the tool of establishing stability with predictive control of nonlinear discretized systems. Here we employ the Lyapunov function to consider the stability of controlled system. We choose a Lyapunov function as:

$$
V_{k}=\left|\mathbf{E}_{m}(k)\right|=\left|\mathbf{X}_{s p}-\mathbf{X}_{m}(k)\right|
$$

So

$$
V_{k+1}=\left|\mathbf{E}_{m}(k+1)\right|=\left|\mathbf{X}_{s p}-\mathbf{X}_{m}(k+1)\right|
$$

Since

$$
\mathbf{U}(k+1)=\Psi_{0}\left(\zeta\left(\mathbf{X}_{m}(k+1), \mathbf{E}(k+1)\right), \eta(k+1)\right)
$$

we can obtain:

$$
\mathbf{X}_{m}(k+1)=\zeta\left(\mathbf{X}_{m}(k+1), \mathbf{E}(k+1)\right)=\varepsilon \mathbf{X}_{m}(k)+(1-\varepsilon)\left[\mathbf{X}_{m}(k+1)+\mathbf{X}_{s p}-\mathbf{X}(k+1)\right]
$$

When $\eta=0$, the Eq.(27) can response the system precisely $\left(\mathbf{X}_{m}(k+1)=\mathbf{X}(k+1)\right)$. And if $\eta \neq 0$ and $|\eta| \leq \rho$, there exist process model errors we can modify the parameter $\varepsilon$ to improve the performance of the controller against the process model errors. So Eq. (32) can be re-written as:

$$
\mathbf{X}_{m}(k+1) \approx \varepsilon \mathbf{X}_{m}(k)+(1-\varepsilon) \mathbf{X}_{s p}
$$

Then

$$
\mathbf{E}_{m}(k+1)=\mathbf{X}_{s p}-\mathbf{X}_{m}(k+1) \approx \varepsilon \mathbf{X}_{s p}-\varepsilon \mathbf{X}_{m}(k) \approx \varepsilon\left(\mathbf{X}_{s p}-\mathbf{X}_{m}(k)\right)
$$


When $\eta=0$, Since $0 \leq \varepsilon \leq 1$, we get:

$V_{k+1}=\left|\mathbf{E}_{m}(k+1)\right|=\varepsilon\left|\mathbf{E}_{m}(k)\right| \leq V_{k}$

If $\eta \neq 0$ and $|\eta| \leq \rho$, we can modify the parameter $\varepsilon$ to make $V_{k+1} \leq V_{k} \cdot \square$

\section{Simulation and Experiment}

\subsection{Simulation}

As a preliminary step before the experimental validation, the control algorithm has been tested by simulation. The sampling time is chosen as $T=0.1 \mathrm{~s}$. First we consider the non-slipping case. Here $\eta(t)=0$. In this case an " 8 " shape reference path is considered. The starting position is chosen as $\left[0.0,0.0,30.0^{\circ}\right]^{T}$. Figure 2 shows the reference path and the tracking performance of the mobile robot by using the MAC tracking control method. The control inputs are shown in Figure 3 and Figure 4 shows the position error $e_{k}=\sqrt{\left(e_{k}^{x}\right)^{2}+\left(e_{k}^{y}\right)^{2}}$ and orientation error $e_{k}^{\theta}$.

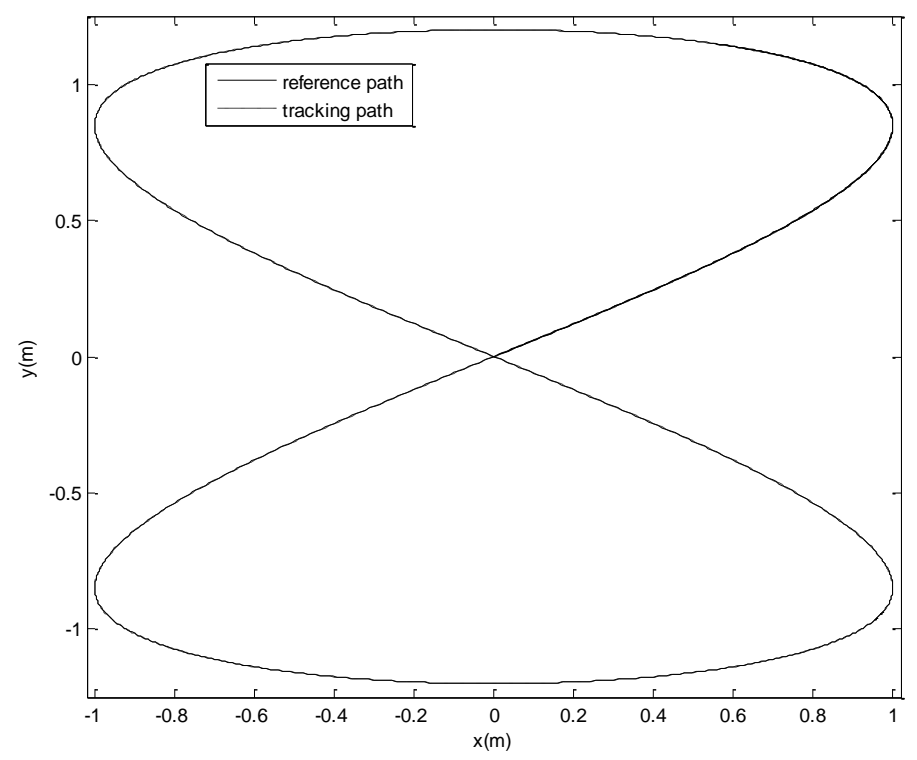

Figure 2. Simulation Result of MAC Tracking Control $(\eta(t)=0)$ 

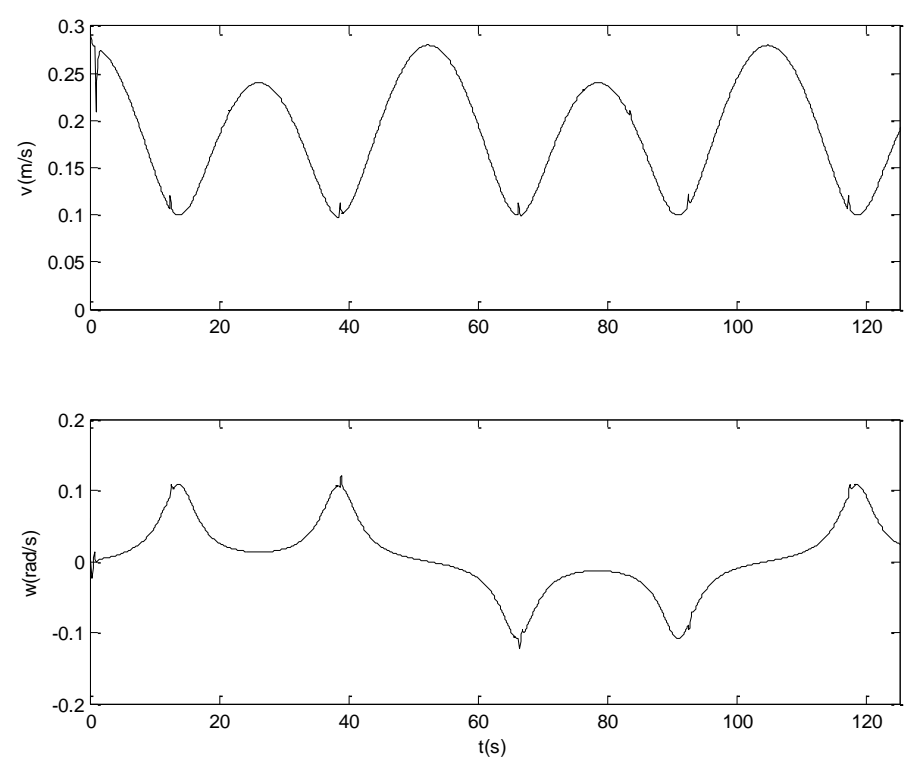

Figure 3. Control Inputs $(\eta(t)=0)$
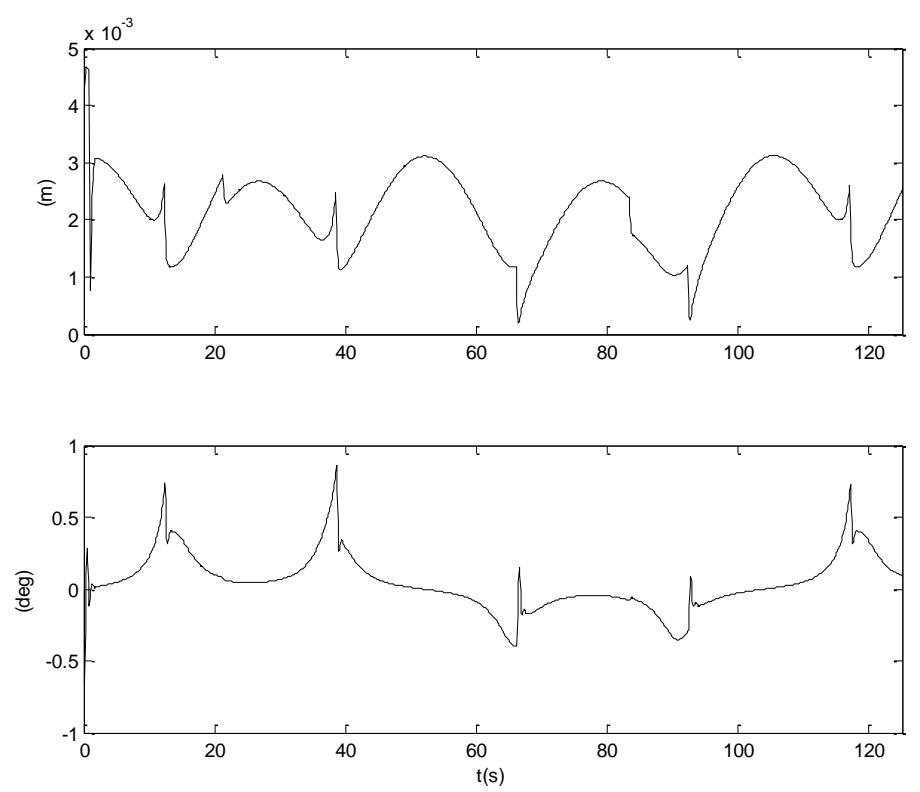

Figure 4. Position Error $e_{k}=\sqrt{\left(e_{k}^{x}\right)^{2}+\left(e_{k}^{y}\right)^{2}}$ and Orientation Error $e_{k}^{\theta}$

$$
(\eta(t)=0)
$$

Then the simulation of MAC tracking control with skidding effect is considered. In this case $\eta(t) \neq 0$. The disturbance $\eta(t)$ used in this simulation is shown in Figure 5 . In this case we also utilize an " 8 " shape reference path to do the simulation. The starting position is chosen as $\left[0.0,0.0,30.0^{\circ}\right]^{T}$. Figure 6 shows the reference path and the tracking performance of the mobile robot by using the MAC tracking method. The control inputs are shown in Figure 7 and Figure 8 shows the position 
error $e_{k}=\sqrt{\left(e_{k}^{x}\right)^{2}+\left(e_{k}^{y}\right)^{2}}$ and orientation error $e_{k}^{\theta}$. The totally calculation time is $0.31 \mathrm{~s}$. From Figure 2-8 we can see that the proposed MAC tracking control method can control the mobile robot to track the reference path accurately even in the case of skidding effects which violate the nonholonomic constraints.

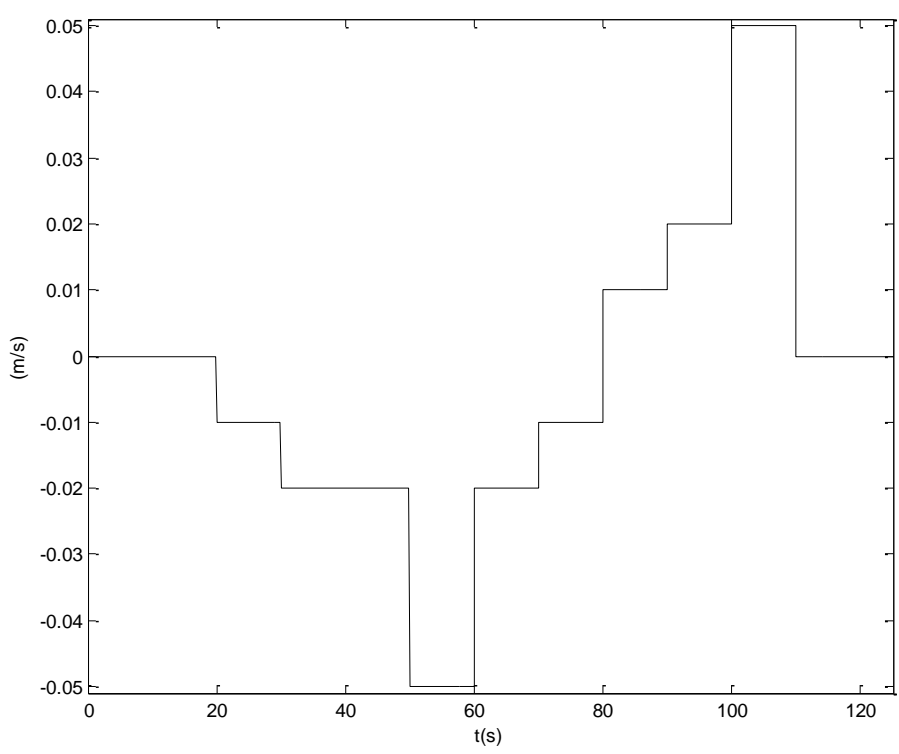

Figure 5. Disturbance $\eta(t)$ for Simulation

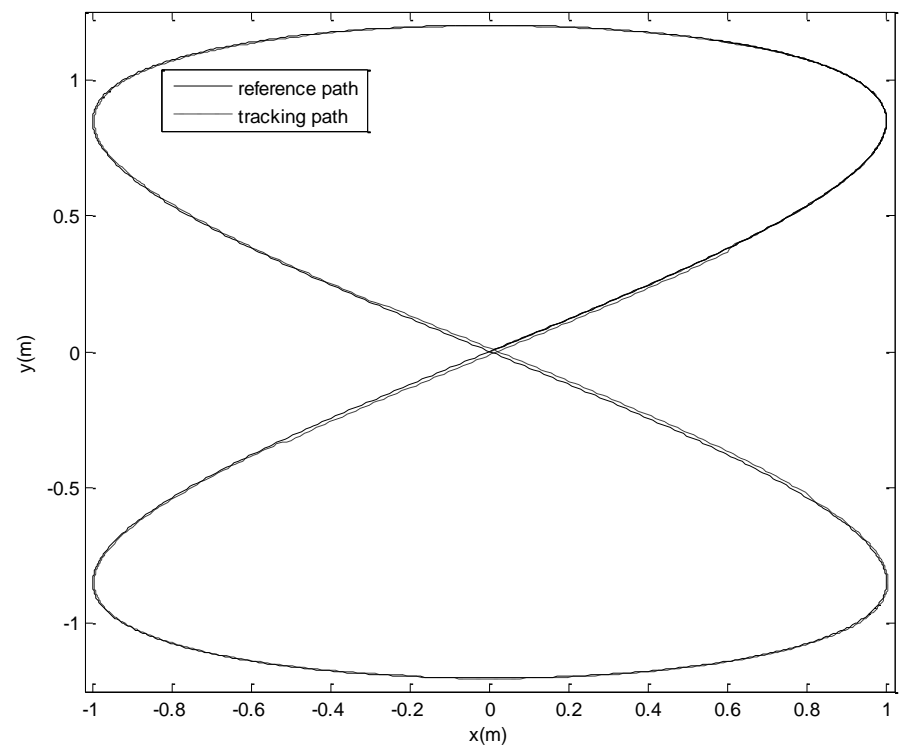

Figure 6. Simulation Result of MAC Tracking Control $(\eta(t) \neq 0)$ 

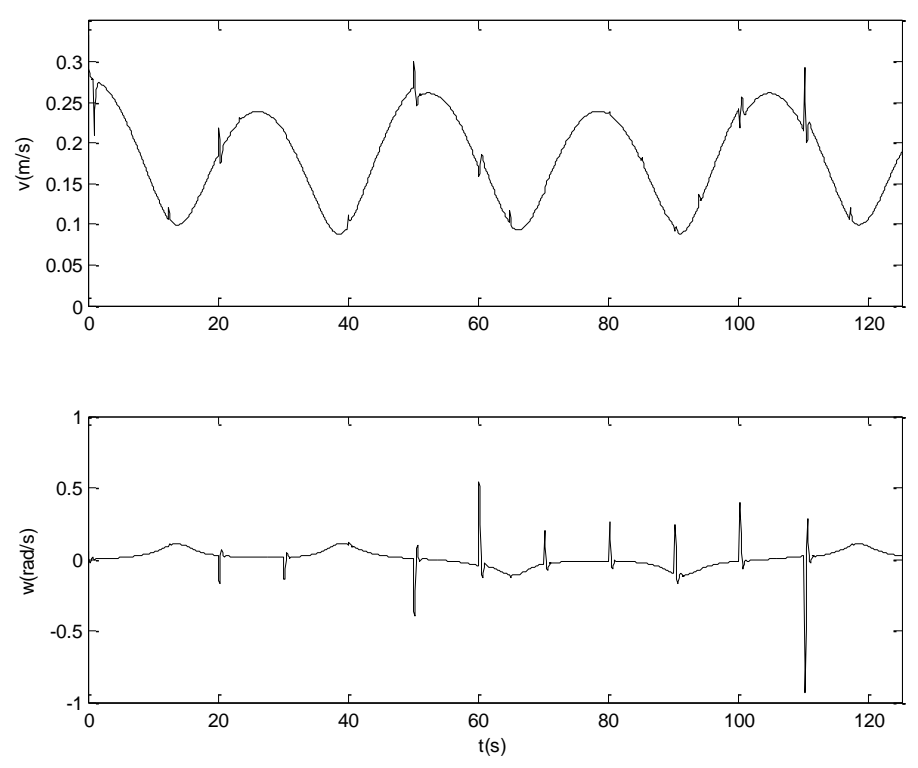

Figure 7. Control Inputs $(\eta(t) \neq 0)$
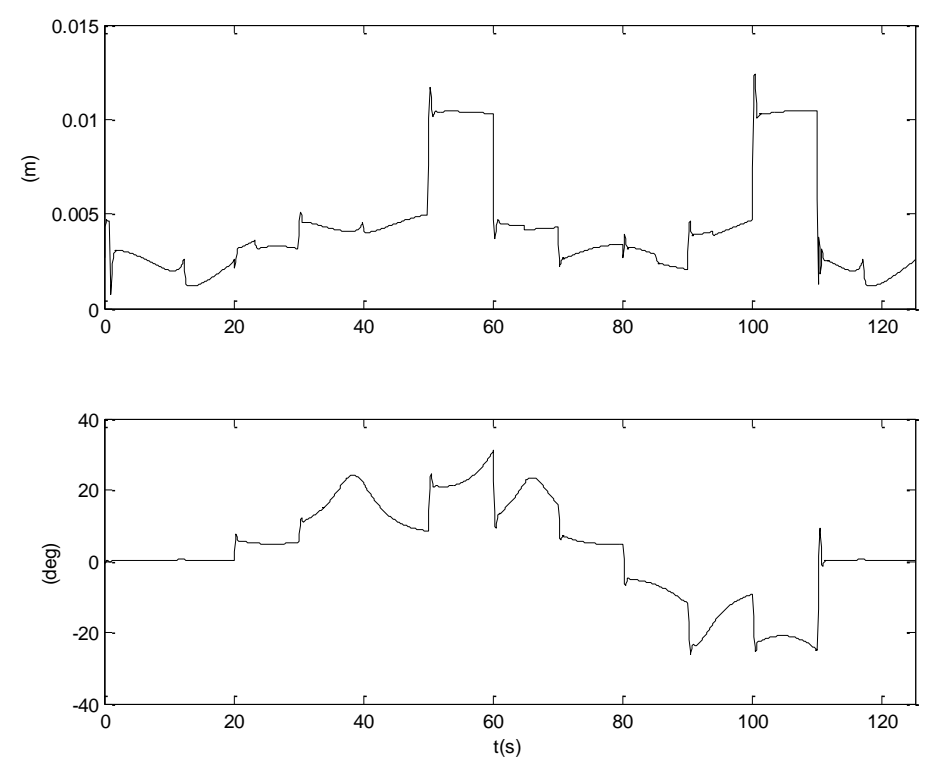

Figure 8. Position Error $e_{k}=\sqrt{\left(e_{k}^{x}\right)^{2}+\left(e_{k}^{y}\right)^{2}}$ and Orientation Error $e_{k}^{\theta}$ $(\eta(t) \neq 0)$

\subsection{Experiment}

The DU ROBO mobile robot of our lab was utilized for the experiment. This mobile robot is supplied with an automatic navigation system. It is a unicycle robot equipped with two driving wheels, DC motors, and it has two encoders and an ecompass to reckon the path and position of the mobile robot. In the experiment the sampling time was also chosen as $T=0.1 \mathrm{~s}$. The disturbance $\eta(t)$ used in the experiment is shown in Figure 9. Figure 10 shows the reference path and the 
tracking performance of the mobile robot by using the MAC tracking method. Figure 11 shows the position error $e_{k}=\sqrt{\left(e_{k}^{x}\right)^{2}+\left(e_{k}^{y}\right)^{2}}$ and orientation error $e_{k}^{\theta}$.

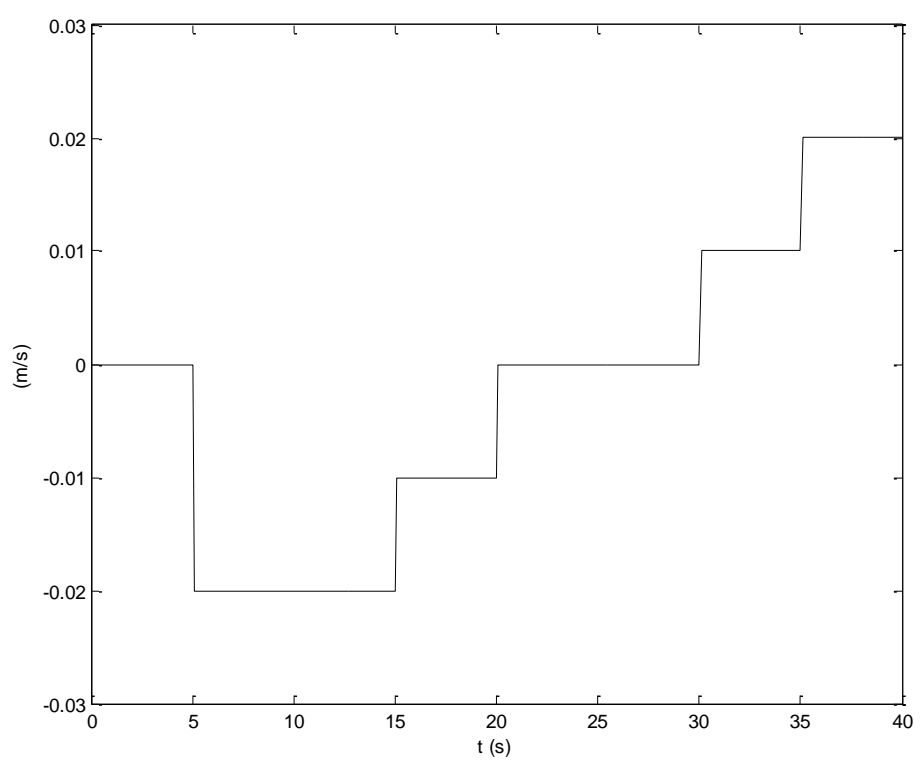

Figure 9. Disturbance for the Experiment

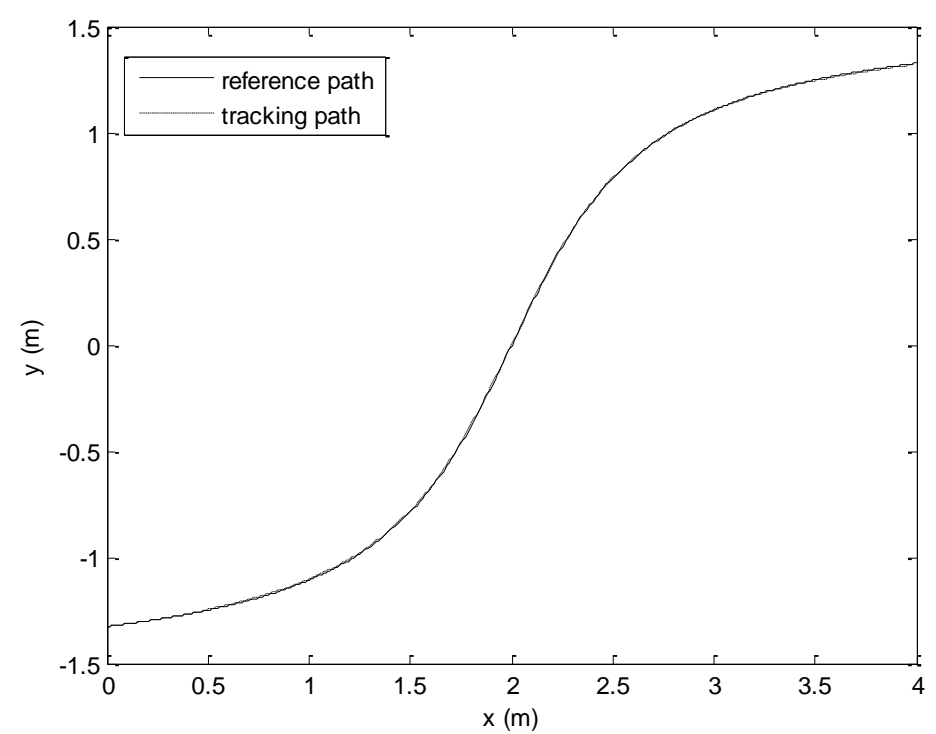

Figure 10. Tracking Performance of the Mobile Robot in the Experiment 

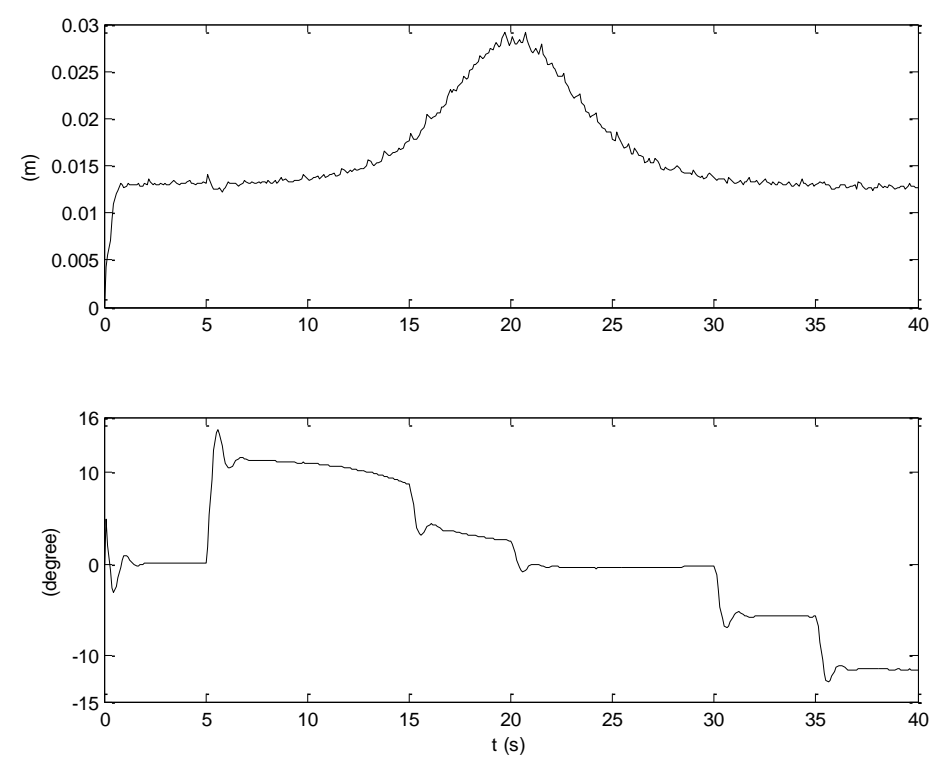

Figure 11. Position Error $e_{k}=\sqrt{\left(e_{k}^{x}\right)^{2}+\left(e_{k}^{y}\right)^{2}}$ and Orientation Error $e_{k}^{\theta}$ in the Experiment

\section{Conclusions}

The path tracking problem of wheeled mobile robots which violates the nonholonomic constraints is studied in this paper. Time discretization method using the Taylor series and the $\mathrm{ZOH}$ assumption was used to obtain the sampled-data representation of the nonlinear WMR's model. The MAC method is proposed to do the tracking control for the mobile robot. The main idea of the MAC strategy is to predict the deviation of the future system outputs from the reference path based on the model, define an optimality criterion that reflects the deviations, and obtain an optimality control strategy to minimize the criterion over a certain horizon in the future. The skidding effect which violates the nonholonomic constraints was considered. Simulations and experiment were conducted to demonstrate the effectivity of the proposed MAC control method in terms of the tracking performance. The good experimental and simulation results showed the potential of the MAC control method for the tracking control of the WMRs.

\section{Acknowledgments}

This paper is supported by Jiangsu Institute of Marine Resources Open Fund Project (JSIMR201407) and the Ministry of Trade, Industry and Energy(MOTIE) and Korea Institute for Advancement of Technology(KIAT) through the International Cooperative R\&D program(N046200012)

\section{References}

[1] K.D. Do, "Bounded Controllers for Global Path Tracking Control of Unicycle-Type Mobile Robots", Robotics and Autonomous Systems, vol. 61, no. 8, (2013), pp. 775-784.

[2] M. Omid, D. Rached and B. R. Ahmad, "Indirect Adaptive Tracking Control of A Nonholonomic Mobile Robot via Neural Networks", Neurocomputing, vol. 88, (2012), pp. 54-66.

[3] J. Huang, C. Wen, W. Wang and Z.-P. Jiang, "Adaptive Output Feedback Tracking Control of A Nonholonomic Mobile Robot”, Automatica, vol. 50, no. 3, (2014), pp. 821-831. 
[4] M. L. Corradini, T. Leo and G. Orlando, "Experimental Testing of a Discrete-Time Sliding Mode Controller for Trajectory Tracking of a Wheeled Mobile Robot in the Presence of Skidding Effects", Journal of Robotic Systems, vol. 19, no. 4, (2002), pp. 177-188.

[5] P. M. George and G. T. Spyros, "Switching Fuzzy Tracking Control for Mobile Robots Under Curvature Constraints", Control Engineering Practice, vol. 19, no. 1, (2011), pp. 45-53.

[6] J. Huang, C. Wen, W. Wang and Z.-P. Jiang, "Adaptive Stabilization and Tracking Control of A Nonholonomic Mobile Robot with Input Saturation and Disturbance”, Systems \& Control Letters, vol. 62, no. 3, (2013), pp. 234-241.

[7] S.G. Tzafestas, K.M. Deliparaschos and G.P. Moustris, "Fuzzy Logic Path Tracking Control for Autonomous Non-Holonomic Mobile Robots: Design of System on a Chip", Robotics and Autonomous Systems, vol. 58, no. 8, (2010), pp. 1017-1027.

[8] Z. R. Cassius, C. Ricardo and S.-F. Mário, "A Nonlinear Trajectory Tracking Controller for Mobile Robots with Velocity Limitation via Fuzzy Gains”, Control Engineering Practice, vol. 21, no. 10, (2013), pp. 1302-1309.

[9] Z. Ilan, A. Amit and R. Raul, "Mobile Robot Characterized by Dynamic and Kinematic Equations and Actuator Dynamics: Trajectory Tracking and Related Application”, Robotics and Autonomous Systems, vol. 59, no. 6, (2011), pp. 343-353.

[10] B. Sašo, "A Novel Trajectory-Tracking Control Law for Wheeled Mobile Robots", Robotics and Autonomous Systems, vol. 59, no. 11, (2011), pp. 1001-1007.

[11] H. Ehsan, G. J. Maani and G. J. Navid, "Model-Based PI-Fuzzy Control of Four-Wheeled OmniDirectional Mobile Robots", Robotics and Autonomous Systems, vol. 59, no. 11, (2011), pp. 930-942.

[12] G. Scaglia, A. Rosales, L. Quintero, V. Mut and R. Agarwal, "A Linear-Interpolation-Based Controller Design for Trajectory Tracking of Mobile Robots", Control Engineering Practice, vol. 18, no. 3, (2010), pp. 318-329.

[13] H. Zenon and S. Marcin, "Discrete Neural Dynamic Programming in Wheeled Mobile Robot Control", Communications in Nonlinear Science and Numerical Simulation, vol. 16, no. 5, (2011), pp. 2355-2362.

[14] H. Lim, Y. Kang, C. Kim and J. Kim, "Experimental Verification of Nonlinear Model Predictive Tracking Control for Six-Wheeled Unmanned Ground Vehicles", International Journal of Precision Engineering and Manufacturing, vol. 15, no. 5, (2014), pp 831-840.

[15] V.-T. Dinh, H. Nguyen, S.-M. Shin, H.-K. Kim, S.-B. Kim and G.-S. Byun, "Tracking Control of Omnidirectional Mobile Platform with Disturbance Using Differential Sliding Mode Controller", International Journal of Precision Engineering and Manufacturing, vol. 13, no. 1, (2012), pp 39-48.

[16] C.-Y. Chen, T.-H. S. Li and Y.-C. Yeh, "EP-Based Kinematic Control and Adaptive Fuzzy SlidingMode Dynamic Control for Wheeled Mobile Robots", Information Sciences, vol. 179, no. 1-2, (2009), pp. 180-195.

[17] R. Gonzalez, M. Fiacchini, T. Alamo, J. L. Guzman and F. Rodriguez, "Adaptive Control for A Mobile Robot Under Slip Conditions Using An LMI-Based Approach”, European Journal of Control, vol. 16, no. 2, (2010), pp. 144-155.

[18] S. J. Yoo, "Adaptive Neural Tracking and Obstacle Avoidance of Uncertain Mobile Robots with Unknown Skidding and Slipping", Information Sciences, vol. 238, (2013), pp. 176-189.

[19] S. X. Yang, A. Zhu, G. Yuan and M.Q.-H. Meng, "A Bioinspired Neurodynamics-Based Approach to Tracking Control of Mobile Robots", IEEE Transactions on Industrial Electronics, vol. 59, no. 8, (2012), pp. 3211-3220.

[20] Y. Gao and K. T. Chong, "Min-Max Model Predictive Controller for Trajectory Tracking of A Wheeled Mobile Robot with Slipping Effects", IEICE Transactions, vol. 94-A, no. 2, (2011), pp. 680-687.

\section{Authors}

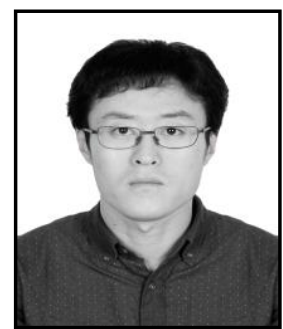

Yuanliang Zhang, graduated from Tsinghua University, China in 2001. He received the M. Sc. and Ph.D. degrees from Chonbuk National University, Korea in 2006 and 2009, respectively. He is currently a postdoctor at School of Automation, Southeast University and an associate professor at School of Mechanical Engineering, Huaihai Institute of Technology, China. His research interests include control system and robotics. 


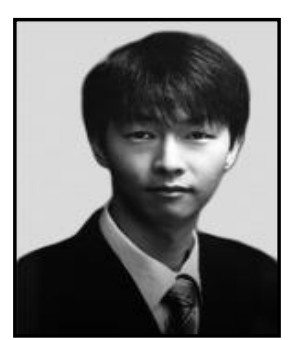

Jihyoung Ryu, graduated from Chonbuk National University, Korea in 2005. He received the M. Sc. and Ph.D. degrees from Chonbuk National University, Korea in 2007 and 2014, respectively. $\mathrm{He}$ is currently a researcher at Electronics and Telecommunications Research Institute, Korea. His research interests include automation system and industrial robotics.

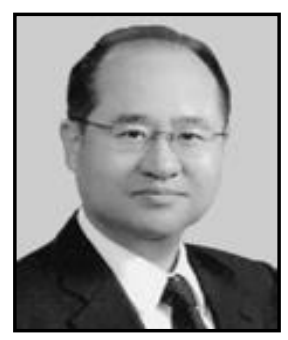

Kil To Chong, received his Ph.D. in Mechanical Engineering from Texas A\&M University in 1995. Currently, he is a professor at the School of Electronics and Information Engineering at Chonbuk National University in Jeonju, Korea, and is head of the Advanced Research Center of Electronics. His research interests are in the areas of signal processing, motor fault detection, network system control, time-delay systems, and neural networks 